\title{
Sex Differences in Atrial Fibrillation
}

\author{
Yasushi Mukai, MD, PhD
}

I $\mathrm{t}$ is well known that there are significant sex differences in the epidemiology of cardiac arrhythmia and electrophysiological features of the myocardium. Numerous previous studies have indicated significant sex differences in the epidemiology, pathophysiology, and therapeutic outcome of atrial fibrillation (AF; Table). ${ }^{1,2}$ In general, AF is more prevalent in men than in women in age-matched observations, and this is also the case in Japan.,3 Conversely, among patients with diagnosed AF, women are more likely to have the persistent form of AF at presentation, heart failure, and stroke, and thus a poorer prognosis. ${ }^{4}$ Importantly, the outcome of AF ablation also differs between men and women, with a higher recurrence rate in women. ${ }^{5}$ The reasons for these clinically important sex differences in AF are not entirely clear; however, it has been reported that women are more likely to have complex proarrhythmic substrates and non-pulmonary vein (PV) triggers of AF. . $^{5-7}$

\section{How Do We Treat Persistent/Recurrent AF in Women?}

In this issue of the Journal, using the database from their previous prospective study, namely the EARNEST-PVI trial, Sato et al tested the hypothesis that an extensive ablation strategy in addition to PV isolation may be more effective for women than men among patients with persistent AF. ${ }^{6}$ Since the hypothesis was unexpectedly rejected based on the study results, the authors concluded that the

\begin{abstract}
Article p????
superiority of an extensive ablation strategy for persistent AF was consistent across both sexes. ${ }^{6}$ As in previous studies, the women undergoing AF ablation were older than the men, tended to have higher $\mathrm{CHA}_{2} \mathrm{DS}_{2}$-VASc scores, and had larger left atrial size considering differences in body height and weight, all of which were related to tougher arrhythmogenic substrates. Most of the extensive ablation groups across men and women underwent additional linear ablations and only few $(<20 \%)$ underwent complex fractionated atrial electrogram (CFAE) ablations. Thus, the favorable outcomes of the extensive ablation groups may generally be attributable to the favorable effect of linear ablations both in men and women. Sato et al speculated that the linear ablations could exert a back-up blocking effect to the PV isolation (PVI) line, ${ }^{6}$ and were thus effective in men even if their atrial arrhythmogenic substrate was not extensive, which may have diminished the differences in the efficacy of extensive ablations between the sexes. Another possibility is that CFAE ablation may have suppressed the arrhythmogenic substrates, especially in women, because CFAE ablation was related to a lower recurrence rate only in women even though the number of cases of CFAE ablation among women was small. The precise reason for the comparable effect of extensive abla-
\end{abstract}

Table. Characteristics of AF in Women Compared With Men
Epidemiology
- Less prevalent than in men in the general population, but increases with advanced age
- More associated with heart failure, as both a predisposing factor and outcome
- Higher incidence of stroke
Pathophysiology
- Anti-arrhythmic estrogen effect on atrial myocardium (less prevalent in younger women)
- A series of pathophysiological and electrical changes after menopause
- Enlarged and fibrotic atria
Electrophysiological study and ablation outcomes
- More complex arrhythmogenic substrate
- More prevalent non-PV AF triggers
- Higher recurrence rate after catheter ablation
AF, atrial fibrillation; PV, pulmonary vein.

The opinions expressed in this article are not necessarily those of the editors or of the Japanese Circulation Society.

Received December 25, 2021; accepted January 4, 2022; J-STAGE Advance Publication released online February 1, 2022

Division of Cardiology, Fukuoka Red Cross Hospital, Fukuoka, Japan

Mailing address: Yasushi Mukai, MD, PhD, FJCS, Division of Cardiology, Fukuoka Red Cross Hospital, 3-1-1 Ogusu, Minamiku, Fukuoka 815-8555, Japan. E-mail: y_mukai@junnai.org

All rights are reserved to the Japanese Circulation Society. For permissions, please e-mail: cj@j-circ.or.jp

ISSN-1346-9843 
tion across both sexes in the study of Sato et al is not clear. It should be noted that no advantages of extensive ablation strategies, especially for women, were reported in subgroup analyses of a previous randomized clinical trial (STAR-AF II) assessing the efficacy of extensive ablation strategies. $^{\mathbf{8}}$

Another important confounding factor in the study of Sato et al is the high prevalence of non-PV triggers in women. Indeed, non-PV triggers were more than threefold more prevalent in women than in men. ${ }^{6}$ Non-PV triggers were allowed to be mapped and ablated in the study, but vigorous induction and mapping of non-PV triggers may be difficult in a procedure when attempting extensive substrate ablations. This may rather favor the PVI-alone group. Indeed, it seems that more patients in the PVI-alone group had documentation and treatment of non-PV triggers among both men and women, which may have affected the study results in both men and women. Finally, it should be again emphasized that no extensive ablation strategy beyond PVI has been established to date for persistent/recurrent AF regardless of sex.

\section{Future Perspectives}

Sex-oriented ablation strategies for AF remain to be established despite significant pathophysiological differences in AF between men and women.9 As suggested in the study of Sato et al, ${ }^{6}$ it may be possible that different kinds of strategies beyond PVI, targeting either triggers or substrates, differently affect clinical outcomes. Future studies are needed to clarify this important issue.

\section{Disclosures}

None declared.

\section{References}

1. Benjamin EJ, Levy D, Vaziri SM, D'Agostino RB, Belanger AJ, Wolf PA. Independent risk factors for atrial fibrillation in a population-based cohort: The Framingham Heart Study. JAMA 1994; 271: 840-844.

2. Odening KE, Deiß S, Dilling-Boer D, Didenko M, Eriksson U, Nedios S, et al. Mechanisms of sex differences in atrial fibrillation: Role of hormones and differences in electrophysiology, structure, function, and remodeling. Europace 2019; 21: 366-376.

3. Akao M, Chun YH, Wada H, Esato M, Hashimoto T, Abe M, et al. Current status of clinical background of patients with atrial fibrillation in a community-based survey: The Fushimi AF Registry. J Cardiol 2013; 61: 260-266.

4. Dagres N, Nieuwlaat R, Vardas PE, Andresen D, Lévy S, Cobbe $\mathrm{S}$, et al. Gender-related differences in presentation, treatment, and outcome of patients with atrial fibrillation in Europe: A report from the Euro Heart Survey on Atrial Fibrillation. J Am Coll Cardiol 2007; 49: 572-577.

5. Kuck KH, Brugada J, Fürnkranz A, Chun KRJ, Metzner A, Ouyang F, et al. Impact of female sex on clinical outcomes in the FIRE AND ICE trial of catheter ablation for atrial fibrillation. Circ Arrhythm Electrophysiol 2018; 11: e006204.

6. Sato T, Sotomi Y, Hikoso S, Nakatani D, Mizuno H, Okada K, et al; on behalf of the Osaka Cardiovascular Conference (OCVC)-Arrhythmia Investigators. Sex differences in the efficacy of pulmonary vein isolation alone vs. extensive catheter ablation in patients with persistent atrial fibrillation. Circ J, doi:10.1253/ circj.CJ-21-0671.

7. Kawai S, Mukai Y, Inoue S, Yakabe D, Nagaoka K, Sakamoto $\mathrm{K}$, et al. Non-pulmonary vein triggers of atrial fibrillation are likely to arise from low-voltage areas in the left atrium. Sci Rep 2019; 9: 12271.

8. Verma A, Jiang CY, Betts TR, Chen J, Deisenhofer I, Mantovan $\mathrm{R}$, et al. Approaches to catheter ablation for persistent atrial fibrillation. N Engl J Med 2015; 372: 1812-1822.

9. Yunus FN, Perino AC, Holmes DN, Matsouaka RA, Curtis AB, Ellenbogen KA, et al. Sex differences in ablation strategy, lesion sets, and complications of catheter ablation for atrial fibrillation: An analysis from the GWTG-AFIB Registry. Circ Arrhythm Electrophysiol 2021; 14: e009790. 